\title{
SOLAR SYSTEM OBJECTS IN THE HIPPARCOS PROGRAMME
}

\author{
A. Bec-Borsenberger \\ Service de Calculs et de Mécanique Céleste \\ du Bureau des Longitudes, URA CNRS 707, \\ 77, Avenue Denfert-Rochereau \\ 75014 Paris, France
}

\begin{abstract}
Forty eight minor planets and two planetary satellites have been retained in the Hipparcos programme. The observations of minor planets will be used to try to determine the dynamical equinox and the rotation of the Hipparcos reference system (S. Söderhjelm and Lindegren, 1982); they can be also used to determine some physical properties of minor planets. As for the observations of planetary satellites, they will allow to obtain accurate ephemerides of these objects and to improve those of the primary planets. The inclusion of minor planets and satellites in the observing programme raises very specific problems: owing to their motion, positions of these objects are given in the Input Catalogue using ephemerides; preparatory astrometric work needed for improving these ephemerides (ground-based observations and improvement of orbital elements) has been described in Bec-Borsenberger $(1989,1991 \mathrm{a})$. To be sure that the ephemerides are sufficient to get the 1 arcsecond predictability needed for Hipparcos, a reduced programme of ground-based observations will last all along the Hipparcos campaign (with the automatic meridian circles of Bordeaux and La Palma observatories). Presently, an annual update is adequate in order to ensure this precision. The improved orbital elements and predicted ephemerides for years 1991 and 1992 are published (Bec-Borsenberger, 1990, 1991b): the residuals obtained from the last set of improved elements with the meridian observations made for the Hipparcos programme give $\mathrm{rms}$ in right ascension and declination between 0.19 and 0.38 aresecond for all Hipparcos minor planets. Since the launch of Hipparcos, the two satellites have been observed and about a third of the minor planets of the programme have been found; the previsions allow to hope to observe this year about ten more minor planets.
\end{abstract}

\section{REFERENCES}

Bec-Borsenberger A.,1989, in ESA-SP 1111, vol. II, 213.

Bec-Borsenberger A., 1990, Ephemerides of the 48 Hipparcos minor planets for the year 1991, Astron. Astrophys. Suppl. Ser. 86, 299.

Bec-Borsenberger A., 1991a, Solar system objects observed by Hipparcos, Astron. Astrophys. (accepted).

Bec-Borsenberger A., 1991b, Ephemerides of the 48 Hipparcos minor planets for the year 1992, Astron. Astrophys. Suppl. (accepted).

Söderhjelm S. and Lindegren L., 1982, Inertial frame determination using minor planets. A simulation of Hipparcos observations, Astron. Astrophys. 110, 156. 